(1)

\title{
Prenatal corticosterone exposure programs sex-specific adrenal adaptations in mouse offspring
}

\author{
J S M Cuffe',2, E L Turton', L K Akison', H Bielefeldt-Ohmann³ and K M Moritz' \\ 1School of Biomedical Science, The University of Queensland, St Lucia, Queensland, Australia \\ 25 chool of Medical Science, Menzies Health Institute Queensland, Griffith University, Gold Coast Campus, \\ Southport, Queensland, Australia \\ ${ }^{3} 5$ chool of Veterinary Science, The University of Queensland, Gatton, Queensland, Australia
}

Correspondence should be addressed to K M Moritz

Email

k.moritz@uq.edu.au

\begin{abstract}
Maternal stress can impair foetal development and program sex-specific disease outcomes in offspring through the actions of maternally produced glucocorticoids, predominantly corticosterone (Cort) in rodents. We have demonstrated in mice that male but not female offspring prenatally exposed to Cort $(33 \mu \mathrm{g} / \mathrm{kg} / \mathrm{h}$ for $60 \mathrm{~h}$ beginning at E12.5) develop cardiovascular/renal dysfunction at 12 months. At 6 months of age, renal function was normal but male offspring had increased plasma aldosterone concentrations, suggesting that altered adrenal function may precede disease. This study investigated the long-term impact of prenatal exposure to Cort on adrenal growth, morphology and steroidogenic capacity as well as plasma Cort concentrations in offspring at postnatal day 30 (PN30), 6 months and 12 months of age. Prenatal Cort exposure decreased adrenal volume, particularly of the zona fasciculata, in male offspring at PN30 but increased both relative and absolute adrenal weight at 6 months of age. By 12 months of age, male Cort-exposed offspring had reduced absolute adrenal weight in association with increased adrenal plaque deposition (lipogenic pigmentation). Plasma Cort concentrations were elevated in male 6-month offspring but not at other ages. mRNA expression of Mc2r (ACTH receptor) was increased in males at PN30, and Cyp11a1 expression was decreased at 6 and 12 months of age. There were no changes in the adrenals of female Cort-exposed offspring. This study demonstrates that prenatal Cort exposure induces offspring adrenal gland dysfunction in an age- and sex-specific manner, which may contribute to long-term programmed disease in male offspring after maternal stress.
\end{abstract} Key Words

Journal of Endocrinology (2017) 232, 37-48

\section{Introduction}

Maternal stress can impair foetal growth and program offspring susceptibility to adult onset disease (Cottrell \& Seckl 2009). Stress-induced impairment of foetal development is mediated by elevated maternal glucocorticoids: cortisol in humans or corticosterone
(Cort) in rodents. Foetal glucocorticoid exposure is necessary for the development of multiple organs (Sugimoto et al. 1976); however, if in excess, glucocorticoids disrupt the formation of physiological systems and program disease in offspring. Much of what

Published by Bioscientifica Ltd. 
we know about glucocorticoid induced programming of disease is based on animal studies using synthetic glucocorticoids such as dexamethasone. Numerous studies have demonstrated that maternal exposure to dexamethasone can reduce foetal or postnatal growth and impair offspring cardiometabolic physiology (Langdown et al. 2001, de Vries et al. 2007, Quinn et al. 2014, Valenzuela et al. 2016). Importantly, this growth restriction is often associated with compensatory catch-up growth, which may further increase the risk of cardiometabolic outcomes (Ozanne 2001). Dexamethasone administration to non-human primates programs hyperinsulinaemia, glucose intolerance and hypertension in offspring along with elevated stress-induced cortisol concentrations (de Vries et al. 2007). This suggests that excess maternal glucocorticoid exposure can program offspring to have perturbed regulation of glucocorticoid production. It is important to note that individuals with excessive glucocorticoid concentrations, such as those with Cushing's disease, have an array of physiological impairments including dysregulated blood pressure, renal function and glucose handling (Anagnostis et al. 2009). Interestingly, males with Cushing's disease display more complex or severe phenotypes than females (Zilio et al. 2014). It has been suggested that programmed alterations in glucocorticoid production may underlie outcomes in models of prenatally programmed disease (Anagnostis et al. 2009).

Production of endogenous glucocorticoids is regulated by the maternal hypothalamic-pituitaryadrenal (HPA) axis. Stress triggers the hypothalamus to produce corticotropin-releasing hormone $(\mathrm{CRH})$, which is secreted into the hypothalamic pituitary portal system to stimulate the anterior pituitary to produce adrenocorticotropic hormone (ACTH). ACTH is released systemically and acts on the melanocortin 2 receptor (MC2R) in the adrenal cortex to drive adrenal growth, development and secretion of glucocorticoids (Chida et al. 2007). Animal studies have demonstrated that disruption of the maternal HPA axis via encephalectomy (Chatelain \& Dupouy 1981) or adrenalectomy (Leret et al. 2004) impairs the development of the foetal adrenal gland. Development of the foetal adrenal gland is regulated by glucocorticoid concentrations as glucocorticoid receptor-knockout mice display poor adrenal gland formation and dysregulated production of ACTH and Cort (Cole et al. 1995).

Although maternal stress has been shown to dysregulate HPA function in offspring (Garcia-Caceres et al. 2010), previous studies have focussed on the hypothalamus or pituitary but not the adrenal gland, despite it being the site of glucocorticoid production. We have previously demonstrated that prenatal Cort exposure in the mouse results in the dysregulation of the renal renin-angiotensin-aldosterone system (RAAS) at 6 months of age (Cuffe et al. 2016) before renal and cardiovascular deficits at 12 months of age (O'Sullivan et al. 2015). Aldosterone levels were elevated in males at 6 months of age suggesting that altered adrenal steroid production may contribute to the impaired physiology observed. This study therefore aimed to use our well-characterised model of prenatal Cort exposure (O'Sullivan et al. 2015) to investigate offspring adrenal gland morphology and function at multiple time points throughout the life course of the animal. As we have previously demonstrated sex-specific differences in foetal and placental growth (Cuffe et al. 2012) as well as disease outcomes after maternal Cort exposure, we investigated adrenal glands from males and females separately.

\section{Methods}

\section{Animal treatment}

All animal experimentation was approved by the University of Queensland Animal Ethics Committee and performed in accordance with the Australian code for the care and use of animals for scientific purposes. Eight-week-old C57BL/6 mice were time mated overnight and housed individually before and throughout pregnancy. At embryonic day 12.5 (E12.5), pregnant mice were anaesthetised with isoflurane for the surgical implantation of an osmotic mini pump primed to release Cort at a dose of $33 \mu \mathrm{g} / \mathrm{kg} / \mathrm{h}$ for $60 \mathrm{~h}$ as described previously (Cuffe et al. 2012). Control mice were left untreated (Untr) to prevent the stress caused by surgery. This treatment regime increases Cort concentrations in the maternal plasma to almost threefold Untr levels (Cuffe et al. 2012). This relative threefold increase in plasma Cort is similar to that seen in male rats exposed to acute restraint stress (Spiers et al. 2016) or in women who have a stressful labour (Van Cauwenberge et al. 1987). Mice were allowed to deliver naturally and were handled regularly by the same personnel to minimise the stress associated with handling. Offspring were culled at postnatal day 30 (PN30), 6 months and 12 months of age for the rapid collection of plasma and adrenal glands. Left adrenals

Published by Bioscientifica Ltd. 
were snap-frozen and stored at $-80^{\circ} \mathrm{C}$ for subsequent gene expression analysis, and right adrenals were fixed in $4 \%$ paraformaldehyde for morphological analysis and immunohistochemical staining.

\section{Plasma Cort measurements}

Cort concentrations were measured at all ages using $10 \mu \mathrm{L}$ of plasma as described in detail previously (Cuffe et al. 2014). Briefly, a radioimmunoassay was used to measure Cort in dichloromethane extracted samples compared to standards using a Cort-specific antibody (Abcam, Cat. No. ab77798). All samples for each age group were run in triplicate within a single assay, and the intra-assay coefficient of variation was $4.35 \%$.
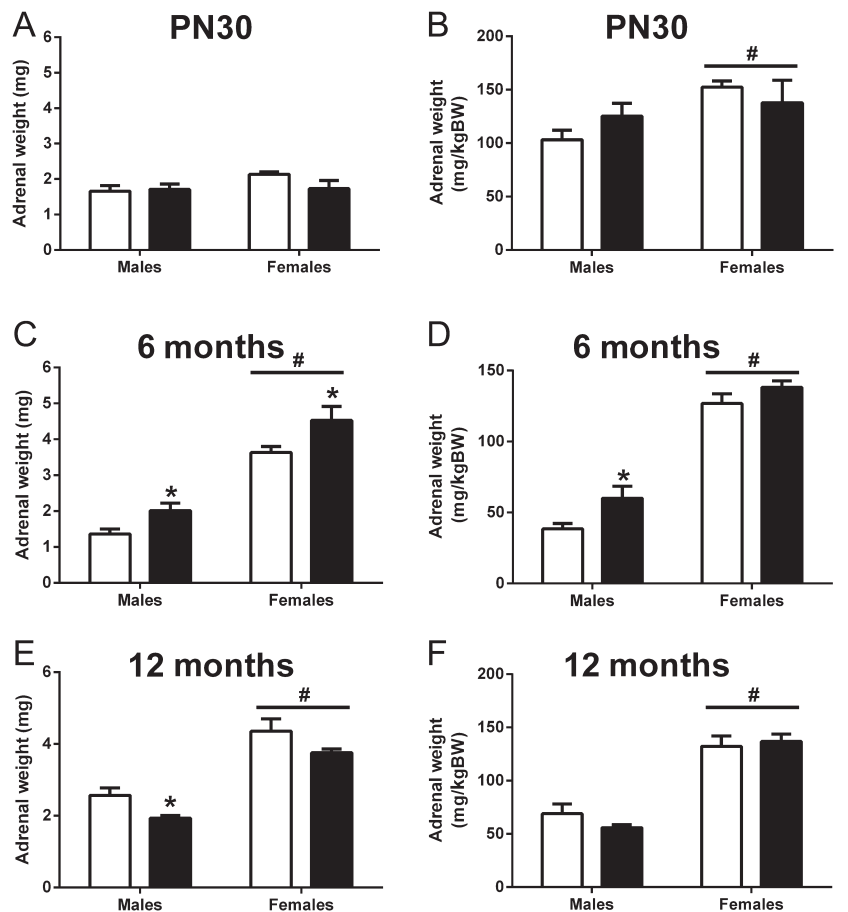

Figure 1

Absolute and relative adrenal weights were increased by prenatal exposure to corticosterone in 6-month-male offspring. The effects of prenatal corticosterone exposure (closed bars) on male and female absolute adrenal weight $(A)$ and relative adrenal weight (B) $(\mathrm{mg} / \mathrm{kgBW}$ ) at postnatal day 30 , absolute adrenal weight (C) and relative adrenal weight (D) at 6 months of age and absolute adrenal weight (E) and relative adrenal weight $(\mathrm{F})$ at 12 months of age compared with untreated control (open bars). Data are represented as means + S.E.M. and analysed using two-way ANOVA with sex and treatment as factors. Sample size - male untreated $=7-9$, male corticosterone $=7-9$, female untreated $=7-13$, female corticosterone $=9-10 . * P<0.05$ used to denote post hoc results when a treatment effect was found and \# used to indicate a sex effect $\left(P_{\text {sex }}<0.05\right)$.

\section{Morphological and stereological analysis}

Fixed adrenal glands were embedded in paraffin and exhaustively sectioned at $7 \mu \mathrm{m}$ and a sampling fraction of 10 (every 6th section) was used to calculate adrenal volume. Remaining slides were kept for immunological analysis. Adrenal sections were stained with a periodic acid Schiff's (PAS) stain to discern medulla and cortical zone regions. Briefly, slides were placed in $1 \%$ periodic acid for $5 \mathrm{~min}$, Schiff's solution for $15 \mathrm{~min}$, haematoxylin for $6 \mathrm{~min}$ and Scott's Tap Water for $2 \mathrm{~min}$. Slides were scanned at 10x magnification using the Aperio Image Scope XT slide scanner (Aperio, Vista, CA, USA) and analysed
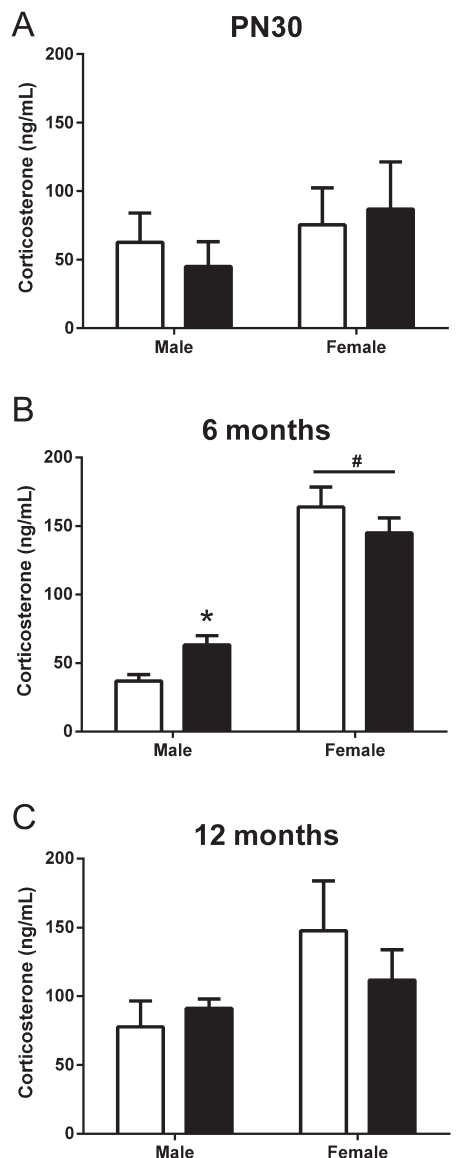

Figure 2

Plasma corticosterone concentrations were increased in 6-month-male offspring after prenatal corticosterone exposure. The effects of prenatal corticosterone exposure (closed bars) on plasma corticosterone concentrations of male and female offspring at (A) postnatal day 30 , (B) 6 months of age and (C) 12 months of age compared with untreated controls (open bars). Data represented as means + S.E.M. and analysed using two-way ANOVA with sex and treatment as factors. Sample size male untreated $=5-8$, male corticosterone $=5-8$, female untreated $=3-5$, female corticosterone $=5-7$. A maximum of 1 animal of each sex was used per litter. ${ }^{*} P<0.05$ used to denote post hoc results when a treatment effect was found and " used to indicate a sex effect $\left(P_{\text {sex }}<0.05\right)$.

Published by Bioscientifica Ltd. 
using the provided software. Images were overlayed with a $1 \mathrm{~cm} \times 1 \mathrm{~cm}$ grid, and the total number of intersecting grid lines were counted for each adrenal region (zona glomerulosa or ZG, zona fasciculata or ZF, zona reticularis or $\mathrm{ZR}$ and medulla or $\mathrm{M}$ ) on each adrenal section. The Cavalieri method was used to estimate medullary and cortical volumes as described previously (Cuffe et al. 2012). As tissue shrinkage occurs in paraformaldehydefixed, paraffin-embedded tissues, values were adjusted for shrinkage using comparative measurements of erythrocyte diameter in fresh and fixed mouse blood.

Pathological analysis of adrenal morphology was assessed by a specialist pathologist who determined that 12-month-old male offspring demonstrated pathological plaque formation including foci of lipogenic pigmentation or 'brown degeneration' (Frith 1983). Follow-up quantification of adrenal plaque number and size was carried out in adrenal glands collected from 12-month-old male offspring. The average number of plaques per adrenal section was analysed on 5 representative midline sections, containing both cortical and medullary tissue, spaced 6 sections apart per adrenal. Adrenal plaques were classified based on plaque size $\left(<101 \mu \mathrm{m}^{2}, 101-500 \mu \mathrm{m}^{2}\right.$, $501-1200 \mu \mathrm{m}^{2}$, and $\left.>1200 \mu \mathrm{m}^{2}\right)$. Counts were separated by region (total cortex and medulla). The area of each section was determined to obtain a total area sampled per adrenal, and all counts were adjusted by a factor proportional to the largest adrenal sampled. Final data were expressed as the number of plaques per section for each adrenal.

\section{Quantitative RT-PCR}

RNA extraction and DNAse digestion were performed on whole adrenal glands using the RNeasy Minikit (Qiagen) following the standard protocol provided. Eluted RNA (200 ng) was reverse transcribed in a $10 \mu \mathrm{L}$ reaction, and $2.5 \mu \mathrm{g}$ of cDNA was used in each reaction. mRNA expression was determined using the comparative CT ( $\left.2^{-\Delta \Delta C T}\right)$ method relative to the geometric mean of $R n 18 \mathrm{~s}$ (Cat\#4319413E, Life Technologies) and beta actin (Actb, Cat\#4352341E). Commercially available TaqMan Assay On Demand primer/probe sets (Life Technologies) were
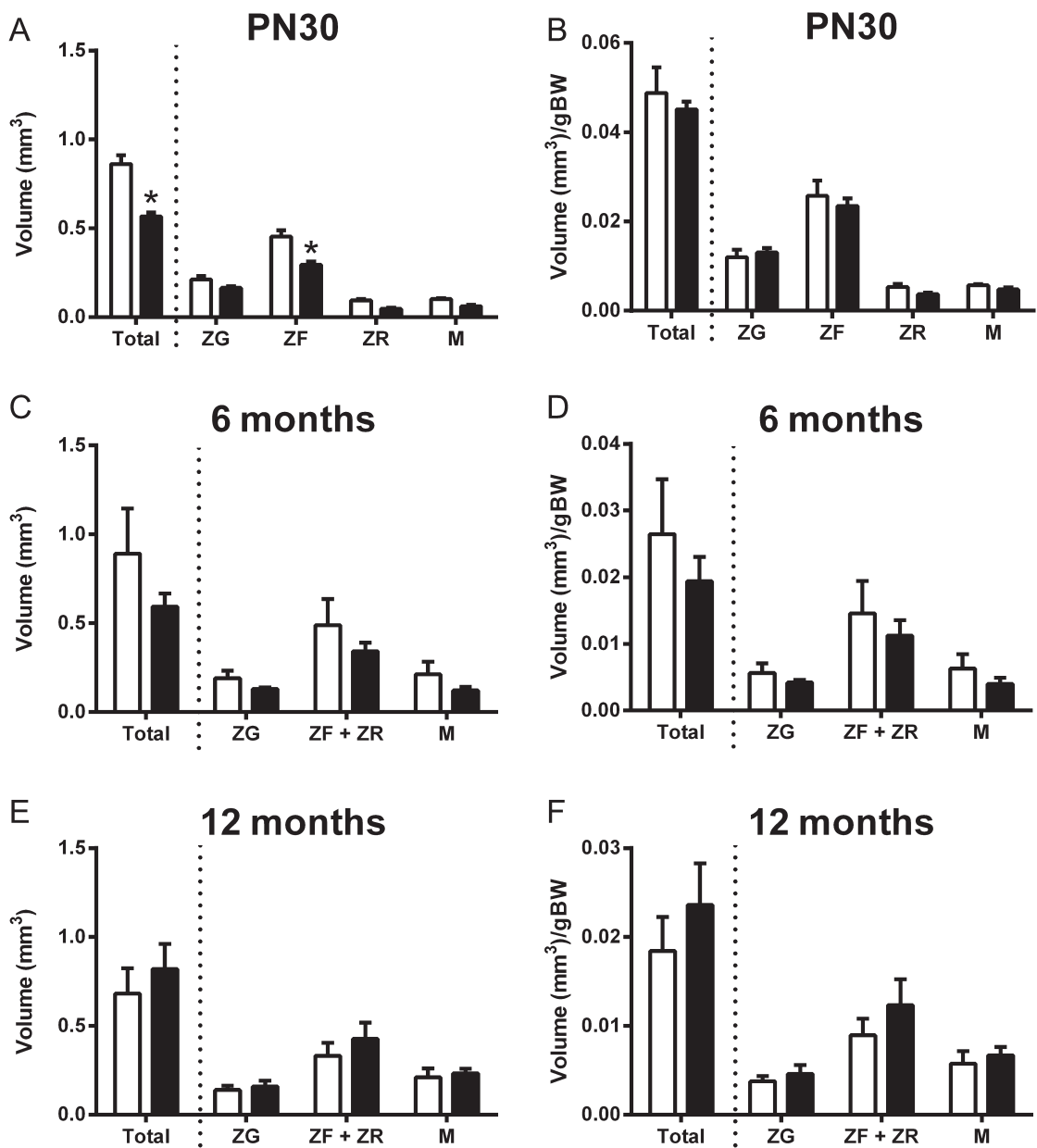
Figure 3
Adrenal volumes were reduced in male offspring at postnatal day 30 . The effects of prenatal corticosterone exposure (closed bars) on absolute adrenal volumes $(\mathrm{A}, \mathrm{C}, \mathrm{E})$ and relative adrenal volumes $\left(\mathrm{mm}^{3} / \mathrm{gBW}, \mathrm{B}, \mathrm{D}, \mathrm{F}\right)$ of male offspring at ( $A$ and $B$ ) postnatal day $30,(C$ and D) 6 months of age and ( $E$ and F) 12 month of age compared with untreated controls (open bars). Adrenal volumes were measured using a stereological approach using every 6 th section throughout the tissue. Sample size - male untreated $=4-5$, male corticosterone $=4$. A maximum of 1 animal was used per litter. Data represented as means+s.E.M. Total volumes and relative volumes were compared between treatment groups using an unpaired Student's $t$-test with $* P<0.05$ used to denote significance. $\mathrm{M}$, medulla; ZF, zona fasciculata; ZG, zona glomerulosa; ZR, zona reticularis.


used to measure mRNA expression of melanocortin 2 receptor (Mc2r, Mm00434865_s1), steroidogenic acute regulatory protein (Star, Mm00441558_m1), cytochrome P450, family 11, subfamily A, polypeptide 1 (Cyp11a1, Mm00490735_m1), cytochrome P450, family 21, subfamily A, polypeptide 1 (Cyp21a1, Mm00487230_g1), cytochrome P450, family 11 , subfamily B, polypeptide 1 (Cyp11b1, Mm01204952_m1) and cytochrome P450, family 11, subfamily B, polypeptide 2 (Cyp11b2, Mm01204955_g1). qPCR reactions were normalised to the mean of the male control group.

\section{Protein localisation}

Protein localisation was assessed using immunohistochemistry for proteins encoded by mRNAs that were affected by prenatal treatment. Representative sections were deparaffinised in xylene and rehydrated through decreasing concentrations of ethanol before undergoing antigen retrieval in citrate buffer at $85^{\circ} \mathrm{C}$. Endogenous peroxidase activity was blocked by immersion in $0.9 \%$ hydrogen peroxide. For MC2R immunolabelling, midline sections of male adrenal glands at PN30 were blocked in $2 \%$ bovine serum albumin before the primary antibody (MC2R polyclonal rabbit, 1:200, H-70, sc-13107, SantaCruz) or an isotype control was applied for an overnight incubation at $4^{\circ} \mathrm{C}$. Slides were incubated with a biotinylated anti-rabbit secondary antibody (ABC Vectastain Elite kit, Vectorlabs, Burlingame, CA, USA) before being exposed to an avidin-biotinylated enzyme complex, stained with $\mathrm{DAB}$ and counterstained with haematoxylin. For CYP11A1 immunolabelling, midline sections of male adrenal glands at 6 and 12 months of age were also blocked by immersion in $0.15 \mathrm{M}$ glycine in PBS for $15 \mathrm{~min}$, followed by $30 \mathrm{~min}$ in DAKO antibody-diluent with background blocker (DAKO) followed by incubation with primary antibody (CYP11A1 polyclonal rabbit, ab175408, Abcam) or rabbit serum in DAKO diluent. Slides were incubated for $2 \mathrm{~h}$ at room temperature followed by visualisation of antibody binding using the DAKO Envision kit for rabbit and AEC chromogen, counterstained with haematoxylin and mounted in DAKO Faramount medium.

\section{Statistical analysis}

All data are expressed as mean \pm S.E.M. and were analysed using GraphPad Prism Software (GraphPad Software). A maximum of 1 adrenal gland of each sex was used for each parameter. Adrenal gland weights, hormones and gene expression were analysed at each time point using two-way analysis of variance (ANOVA) with treatment and sex as factors. Gene expression data were normalised to the average of the Untr male group at each time
12 months
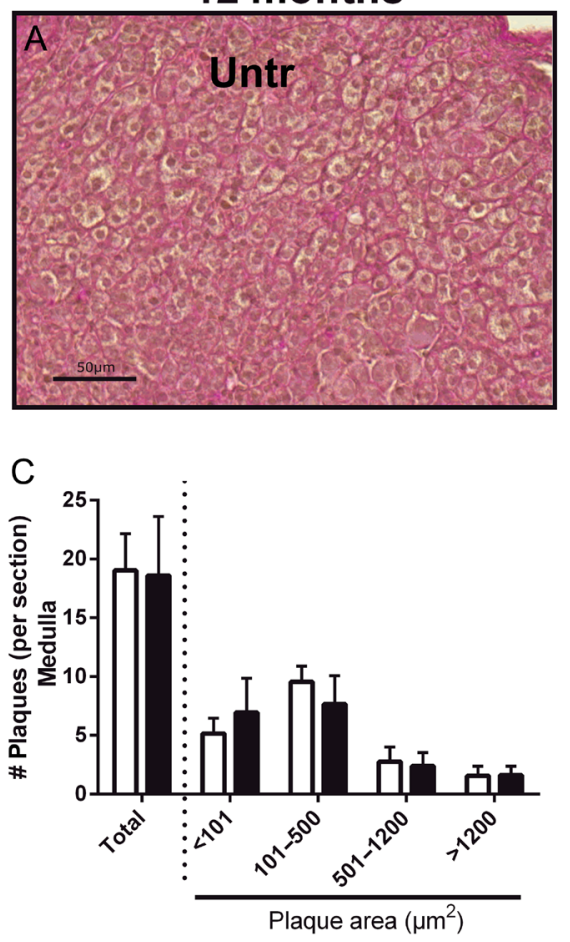

12 months
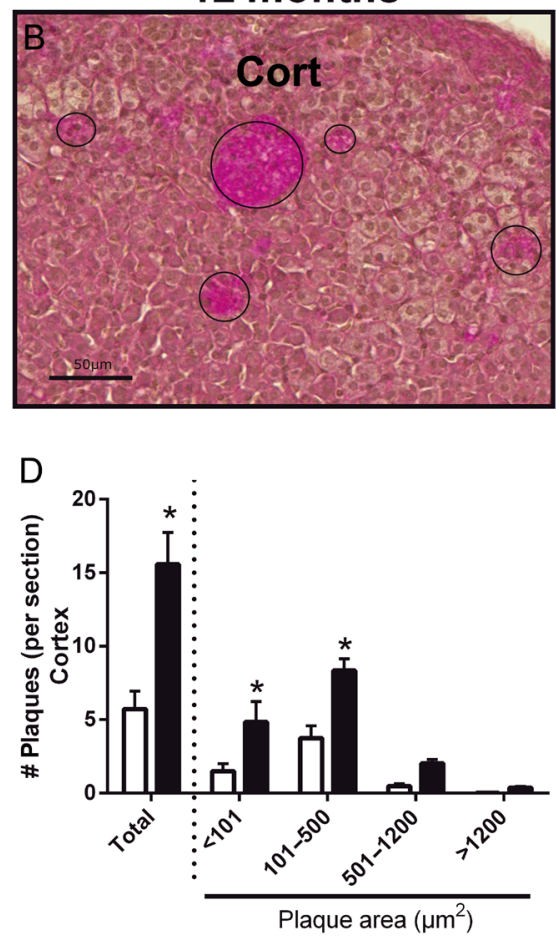

\section{Figure 4}

Morphological analysis demonstrates increased plaque formation in 12-month-old male offspring. Periodic Acid Schiff's stained adrenal glands at $10 \times$ magnification of (A) untreated and (B) corticosterone-exposed 12-month offspring demonstrating an increased number of adrenal plaques (circled areas) in the cortex (D) but not medulla (C) in response to prenatal corticosterone exposure (Scale bars $=50 \mu \mathrm{m}$ ). Plaque number was assessed using 5 representative midline sections, spaced 6 sections apart per adrenal. Sample size male untreated $=5$, male corticosterone $=4$. A maximum of 1 animal was used per litter. Data represented as means + S.E.M., total plaque number and plaques of various sizes were compared between groups using an unpaired Student's $t$-test with $* P<0.05$ used to denote significance. A full colour version of this figure is available at http://dx.doi.org/10.1530/ JOE-16-0417. 
point. Post hoc analysis was performed when required. Total and regional adrenal volumes and plaque numbers were compared using unpaired Student's t-tests. Welch's correction was used when groups to be compared had unequal variances.

\section{Results}

\section{Offspring adrenal weights}

The absolute adrenal gland weight of Untr male offspring was similar between PN30 and 6 months of age and increased in size between 6 months of age and 12 months of age $(P<0.05)$. In contrast, adrenal weight increased from PN30 to 6 months of age in female offspring $(P<0.05)$ but was not significantly greater at 12 months of
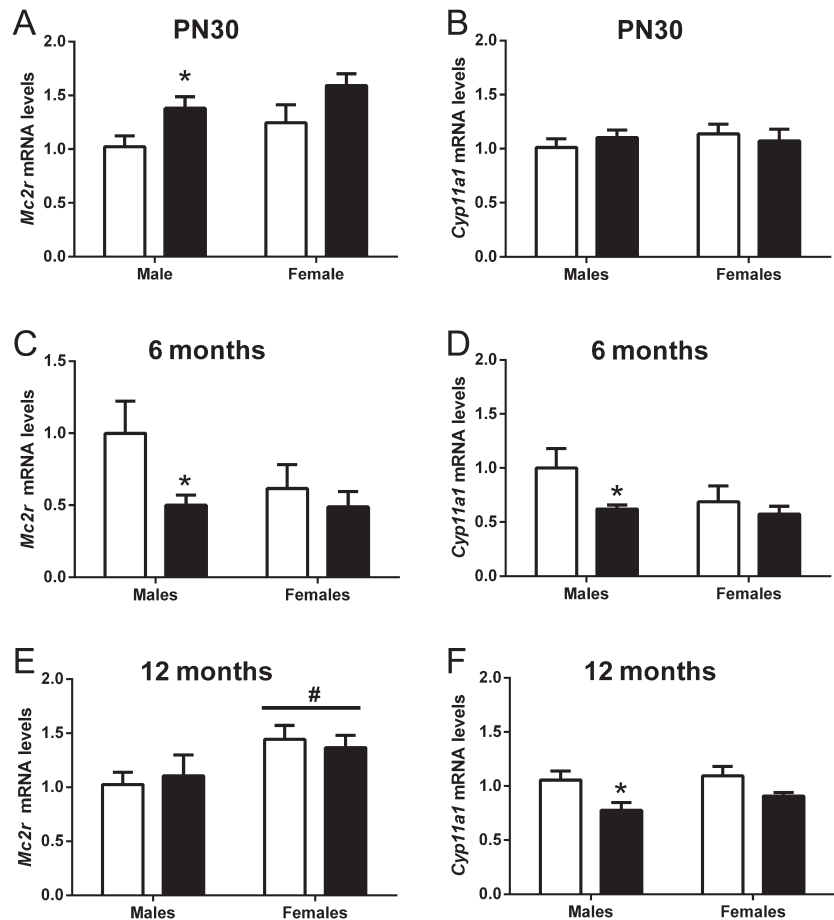

Figure 5

Mc2r and Cyp11a1 were dysregulated in corticosterone-exposed male adrenals. The effects of prenatal corticosterone exposure (closed bars) on relative $M c 2 r$ mRNA expression at (A) postnatal day 30, (C) 6 months of age and (E) 12 months of age and relative Cyp11a1 mRNA expression at (B) postnatal day 30, (D) 6 months of age and (F) 12 months of age compared with untreated controls (open bars). All data are normalized to the male control group of each age. Sample size - male untreated $=6-9$, male corticosterone $=6-9$, female untreated $=6-8$, female corticosterone $=6-9$. A maximum of 1 animal of each sex was used per litter. Data represented as means + S.E.M. and analysed using two-way ANOVA with sex and treatment as factors. $* P<0.05$ used to denote post hoc results when a treatment effect was found and \# used to indicate a sex effect $\left(P_{\text {sex }}<0.05\right)$. age (Fig. 1). Absolute adrenal weight was not affected by prenatal Cort exposure or the sex of the offspring at PN30 (Fig. 1A). The adrenal to body weight ratio at PN30 was also not affected by prenatal Cort exposure but was greater in females compared with males ( $P_{\text {sex }}<0.05$, Fig. 1B). By 6 months of age, female offspring had heavier adrenal glands than males both in terms of absolute adrenal weight $\left(P_{\text {sex }}<0.05\right.$, Fig. 1C) and adrenal-to-body weight ratio $\left(P_{\text {sex }}<0.05\right.$, Fig. 1D). Prenatal Cort exposure was found to increase both absolute adrenal weight $\left(P_{\mathrm{trt}}<0.05\right.$, Fig. 1C) and relative adrenal weight $\left(P_{\text {trt }}<0.05\right.$, Fig. 1D) in 6-month-old offspring. Post hoc analysis demonstrated that absolute adrenal weight was increased in both male and female 6-month-old offspring ( $P<0.05$, Fig. 1C) and relative adrenal weight was increased in male 6-month offspring by prenatal Cort exposure $(P<0.05$, Fig. 1D). Adrenal glands of female offspring remained heavier than male offspring at 12 months of age $\left(P_{\text {sex }}<0.05\right.$, Fig. $1 \mathrm{E}$ and F). Maternal Cort exposure decreased absolute adrenal weights in 12-month-old offspring $\left(P_{\text {trt }}<0.05\right.$, Fig. 1E), but relative adrenal gland weights were unaffected by prenatal treatment (Fig. 1F).

\section{Plasma hormones}

At PN30, plasma Cort concentrations were unaffected by prenatal treatment or the sex of the offspring (Fig. 2A). However, by 6 months of age, females had higher plasma concentrations of Cort than males $\left(P_{\text {sex }}<0.05\right.$, Fig. 2B). Although Cort concentrations were not increased overall by prenatal treatment, a sex by treatment interaction $\left(P_{\text {int }}<0.05\right)$ and post hoc analysis $(P<0.05)$ demonstrated that plasma Cort concentrations in male offspring were increased by $70 \%$ in Cort-exposed offspring compared with Untr controls. By 12 months of age, plasma Cort concentrations no longer differed between prenatal treatment groups or sexes (Fig. 2C).

\section{Adrenal volumes and morphology}

Given that adrenal weights and plasma Cort concentrations were only affected in male offspring, total adrenal and compartment volumes were assessed in male offspring only. Total adrenal volume at PN30 was reduced by approximately 35\% $(P<0.01$, Fig. 3A); however, when normalised to body weight, this reduction in volume was lost (Fig. 3B). Further analysis demonstrated that the overall reduction in absolute adrenal volume was due to a significantly smaller zona

Published by Bioscientifica Ltd 
fasciculata in the adrenals of Cort-exposed offspring compared with controls $(P<0.05)$. Neither the absolute nor relative volume of the adrenal gland was affected by prenatal treatment at either 6 months or 12 months of age (Fig. 3C, D, E and F). Adrenal glands from males and females at PN30 and 6 months demonstrated typical cellular morphology, with defined cortical zones and a medullary region. However, by 12 months of age, adrenal glands from males demonstrated large, anucleated eosinophilic areas, which were also PAS positive and identified as lipogenic pigmentation by an expert pathologist (Fig. 4A and B). These 'plaques' were quantified, and male offspring prenatally exposed to Cort had a greater overall number of these adrenal 'plaques' in the cortex but not in the medulla $(P<0.05)$. This was due to an increase in smaller plaques $\left(<101 \mu \mathrm{m}^{2}\right.$ and $\left.101-500 \mu \mathrm{m}^{2}\right)$ in Cort-exposed offspring compared with Untr control $(P<0.0001$, Fig. $4 \mathrm{C}$ and D).

\section{Gene expression}

Maternal Cort exposure programmed an increase in $M c 2 r$ mRNA expression at PN30 compared with Untr offspring $\left(P_{\text {trt }}<0.05\right.$, Fig. 5A). Post hoc analysis demonstrated that this was due to a $35 \%$ increase in $M c 2 r$ gene expression in male offspring $(P<0.05) . M c 2 r$ mRNA expression was reduced in 6-month offspring prenatally exposed to Cort $\left(P_{\text {trt }}=0.05\right.$, Fig. 5C) with post hoc analysis demonstrating that $M c 2 r$ mRNA expression was reduced by $\sim 50 \%$ in male Cort offspring compared with that in Untr offspring $(P<0.05)$. $M c 2 r$ mRNA expression was not affected by prenatal Cort exposure at 12 months of age but was higher overall in females compared with males $\left(P_{\text {sex }}<0.05\right.$, Fig. 5E). Cyp11a1 mRNA expression was unaffected by prenatal treatment or sex of the offspring at PN30 (Fig. 5B) but was reduced by prenatal Cort exposure at 6 months of age $\left(P_{\text {trt }}<0.05\right.$, Fig. 5D). Post hoc analysis demonstrated that Cyp11a1 gene expression was reduced by $\sim 40 \%$ in male offspring at 6 months of age $(P<0.05)$. Cyp11a1 mRNA expression remained lower at 12 months of age in Cort-exposed offspring compared with Untr controls $\left(P_{\text {trt }}<0.05\right.$, Fig. 5F) with post hoc analysis demonstrating that this reduction was significant in male offspring $(P<0.05)$. Star, Cyp11b1, Cyp11b2 and $C y p 21$ were all unaffected by prenatal treatment at any age (Table 1). However, the expression of some factors was affected by sex with Star being lower in females compared with that in males at 6 months $\left(P_{\text {sex }}<0.05\right)$, $C y p 11 b 1$ higher in females than males at 12 months $\left(P_{\text {sex }}<0.05\right)$, Cyp11b2 lower in females than males at PN30 and Cyp21 higher in females than males at both PN30 $\left(P_{\text {sex }}<0.05\right)$ and at 12 months $\left(P_{\text {sex }}<0.05\right)$ of age (Table 1).

Table 1 Relative mRNA expression for steroidogenic enzymes in the adrenal cortex. Gene expression was measured in adrenal glands collected from male and female offspring prenatally exposed to corticosterone (Cort) or untreated (Untr) and is expressed relative to Rn18s and Actb expression. All data are normalised to the male control group of each age. A maximum of 1 animal of each sex was used per litter. Sample sizes are shown in brackets. PN30=postnatal day 30. Data represented as means + S.E.M. and analysed using a two-way ANOVA with sex and treatment as factors. Only sex differences were detected with post analysis identifying specific sex differences within treatment groups which are annotated with \# when $P<0.05$.

\begin{tabular}{|c|c|c|c|c|}
\hline & \multicolumn{2}{|c|}{ Male } & \multicolumn{2}{|c|}{ Female } \\
\hline & Untr (6-9) & Cort (6-9) & Untr (6-8) & Cort (6-9) \\
\hline \multicolumn{5}{|l|}{ Star } \\
\hline PN30 & $0.97 \pm 0.09$ & $1.22 \pm 0.17$ & $1.07 \pm 0.15$ & $1.21 \pm 0.17$ \\
\hline 6 months & $1.00 \pm 0.15$ & $0.81 \pm 0.07$ & $0.63 \pm 0.15^{\#}$ & $0.45 \pm 0.10^{\#}$ \\
\hline 12 months & $1.09 \pm 0.15$ & $0.72 \pm 0.08$ & $0.97 \pm 0.07$ & $0.93 \pm 0.07$ \\
\hline \multicolumn{5}{|l|}{ Cyp11b1 } \\
\hline PN30 & $1.01 \pm 0.11$ & $1.13 \pm 0.15$ & $1.28 \pm 0.16$ & $1.28 \pm 0.17$ \\
\hline 6 months & $1.34 \pm 0.35$ & $0.98 \pm 0.04$ & $2.02 \pm 0.0 .71$ & $1.34 \pm 0.42$ \\
\hline 12 months & $1.04 \pm 0.11$ & $0.90 \pm 0.09$ & $2.28 \pm 0.28^{\#}$ & $2.19 \pm 0.10^{\#}$ \\
\hline \multicolumn{5}{|l|}{ Cyp11b2 } \\
\hline PN30 & $1.32 \pm 0.22$ & $1.86 \pm 0.21$ & $1.41 \pm 0.27^{\#}$ & $1.00 \pm 0.16^{\#}$ \\
\hline 6 months & $1.38 \pm 0.38$ & $0.87 \pm 0.17$ & $0.63 \pm 0.17$ & $0.63 \pm 0.14$ \\
\hline 12 months & $1.56 \pm 0.36$ & $1.13 \pm 0.26$ & $1.17 \pm 0.26$ & $0.53 \pm 0.16$ \\
\hline \multicolumn{5}{|l|}{ Cyp21 } \\
\hline PN30 & $1.02 \pm 0.14$ & $1.14 \pm 0.14$ & $1.62 \pm 0.17^{\#}$ & $1.51 \pm 0.20^{\#}$ \\
\hline 6 months & $1.29 \pm 0.06$ & $1.66 \pm 0.08$ & $1.54 \pm 0.55$ & $1.24 \pm 0.40$ \\
\hline 12 months & $1.05 \pm 0.14$ & $0.99 \pm 0.16$ & $1.86 \pm 0.05^{\#}$ & $1.79 \pm 0.10^{\#}$ \\
\hline
\end{tabular}

http://joe.endocrinology-journals.org DOI: 10.1530/JOE-16-0417
(C) 2017 Society for Endocrinology Printed in Great Britain
Published by Bioscientifica Ltd 


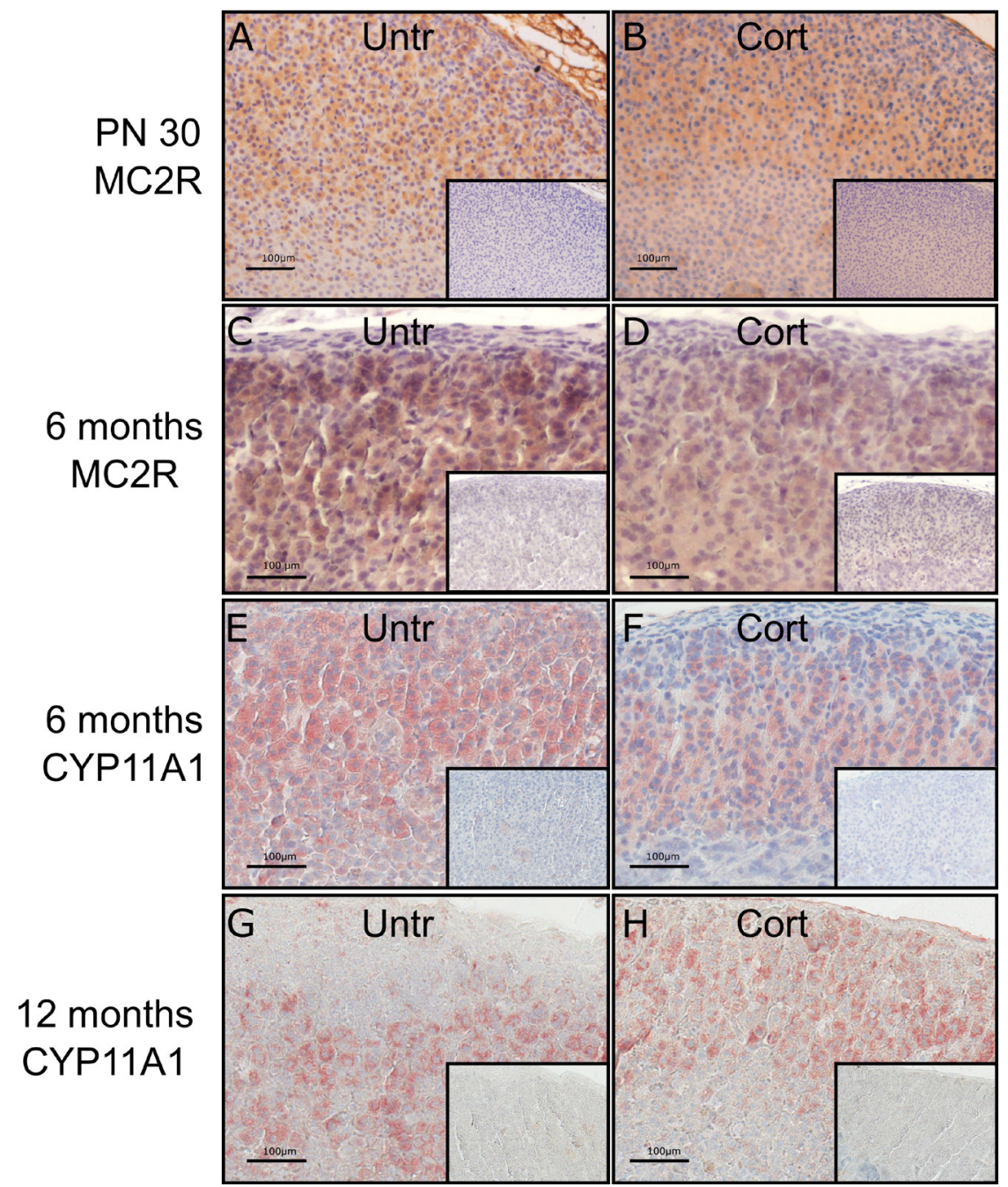

\section{Figure 6}

Immunohistochemical staining demonstrates that corticosterone exposure dysregulates morphology and protein localisation in adrenal glands of male offspring. Immunohistochemical staining of MC2R in untreated $(A)$ and corticosterone (B)-exposed adrenal glands at PN30 (DAB chromogen) and untreated (C) and corticosterone (D)-exposed adrenal glands at 6 months (AEC chromogen) of age, CYP11A1 in untreated (E) and corticosterone (F)-exposed adrenal glands at 6 months of age (AEC chromogen) and CYP11A1 in untreated (G) and corticosterone $(\mathrm{H})$-exposed adrenal glands at 12 months of age (AEC chromogen). Inset images are from negative controls in which the primary antibody was replaced with an isotype control. Scale bars $=100 \mu \mathrm{m}$, Untreated labelled as Untr and Corticosterone labelled as Cort. $n=3-5$ per group with at least two sections stained per adrenal. A maximum of 1 animal was used per litter.

\section{Protein localisation}

Given that $M c 2 r$ mRNA expression was increased in whole adrenal glands of male offspring at PN30 but decreased at 6 months and Cyp11a1 was decreased at both 6 and 12 months, immunohistochemistry was performed in histological sections of the corresponding animals. MC2R protein expression at PN30 was found to be predominantly localised to the zona fasciculata with less expression found in the zona glomerulosa and minimal staining in the zona reticularis and adrenal medulla (Fig. 6A and B). At 6 months of age, MC2R immunostaining was greatest within the zona fasciculata but was also present within the zona glomerulosa and zona reticularis (Fig. 6C and D). CYP11A1 immunohistochemical staining was localised to the zona fasciculata and zona glomerulosa in an age- and treatment-dependent manner. At 6 months of age, CYP11A1 was predominantly localised to the zona fasciculata (Fig. 6E and F). At 12 months of age, CYP11A1 was predominantly localised to the zona fasciculata in Untr offspring with minimal staining in the zona glomerulosa. In contrast, CYP11A1 was predominantly expressed in the zona glomerulosa in Cort-exposed offspring (Fig. 6G and H).

\section{Discussion}

Maternal glucocorticoid exposure is known to impair foetal HPA axis development and program long-term disease. Although there is evidence of a dysregulated HPA in offspring after prenatal endogenous glucocorticoid exposure, little is known about adrenal morphology and function and rarely have offspring of both sexes been examined. This study highlights that female offspring have larger adrenal glands, higher expression of key steroidogenic factors and higher plasma Cort concentrations but that none of these measurements were

Published by Bioscientifica Ltd. 
affected by prenatal Cort exposure. In contrast, in males, prenatal Cort exposure altered adrenal growth and gene expression across the lifespan. Male offspring of dams exposed to Cort during pregnancy had higher plasma concentrations of Cort at 6 months of age which was before the development of the disease previously reported (O'Sullivan et al. 2015, Cuffe et al. 2016). This increase in plasma Cort concentration likely reflects the increase in adrenal size at this age. In addition, changes to $M c 2 r$ and Cyp11a1 gene expression may be contributing to the programmed changes in adrenal size that occur over the life course of the animal and therefore may be indirectly contributing to the increase in plasma Cort concentrations at 6 months of age. Along with the increased adrenal 'plaque' formation, which is associated with ageing, it is likely that the adrenal deficits have contributed to the renal and cardiovascular phenotypes previously reported in 12-month Cort-exposed male offspring.

Although prenatal Cort exposure has altered the growth of the adrenal glands, these outcomes vary with age suggesting that they may be adaptive rather than progressive. We have previously demonstrated that prenatal Cort exposure results in reduced body weight at PN30 (O'Sullivan et al. 2015) but normal body weight by 6 months of age (Cuffe et al. 2016) indicating catch-up growth, a phenomenon often associated with long-term disease propensity (Huxley et al. 2000). In this study, although adrenal weight was unaffected by prenatal treatment at PN30, by 6 months of age, adrenal glands of male offspring exposed to Cort were heavier, suggesting accelerated adrenal growth that is greater than the animal growth over this time. It is important to note that although absolute adrenal weight in female offspring increased significantly from PN30 to 6 months even in control animals, adrenal weight in male animals remained consistent over this period as did Cort levels. These sex differences in adrenal growth have been documented previously in mice (Raber et al. 2000, Bielohuby et al. 2007) and may contribute to the differences in disease vulnerability between males and females. Indeed, we have recently demonstrated in the rat that prenatal stress induces sex- and age-specific dysregulation of androgenregulated genes in the adrenal gland (Cheong et al. 2016).

At PN30 and 6 months, plasma Cort concentrations reflected relative adrenal weight with no differences at PN30 but a 70\% increase in plasma Cort at 6 months of age. In contrast, maternal dexamethasone exposure in spiny mice had no effect on relative adrenal weight or offspring cortisol production (Quinn et al. 2014), whereas maternal dexamethasone administration in rats programmed elevated plasma Cort concentrations in offspring, without affecting relative adrenal weights (Waddell et al. 2010). Conversely, dexamethasone administration in the guinea pig increased the relative adrenal weight but not cortisol concentrations in offspring (Banjanin et al. 2004). These differences likely reflect glucocorticoid- and speciesspecific effects (Singh et al. 2012) but in all these studies, only a single offspring time point was investigated. This study highlights temporal changes in the adrenal glands of offspring and the emergence of specific abnormalities with ageing as evidenced by the fact that at 12 months of age, male offspring of Cort-exposed dams had an increased number of adrenal plaques. These deposits have been shown to be associated not only with ageing (Rosol et al. 2001) but also elevated corticosteroid levels (Schardein et al. 1967, Dunn 1970, Dillberger et al. 1992).

In addition to changes in adrenal growth, the expression of factors, which regulate adrenal growth and function were dysregulated in an age- and sexspecific manner. Mc2r mRNA expression was increased in male offspring prenatally exposed to Cort at PN30 but decreased in male offspring at 6 months of age. ACTH acts on MC2R, located primarily within the zona fasciculata, to increase adrenal cell volume (Ferreira et al. 2007) and drive glucocorticoid production (Chida et al. 2007). It is likely that the observed increase in $M c 2 r$ at PN30 has driven adrenal growth up to 6 months of age. In turn, this increase in adrenal size at 6 months of age may have contributed to the increased production of Cort as well as aldosterone (Cuffe et al. 2016). The decrease in $M c 2 r$ expression at 6 months may be a compensatory adaptation to the increase in plasma Cort concentration initiated through negative feedback, which may have later resulted in the normalisation in adrenal size seen at 12 months of age. Interestingly, studies in guinea pigs have demonstrated that prenatal stress (Kapoor et al. 2008) but not dexamethasone (Banjanin et al. 2004) programmed an increase in Mc2r mRNA expression in offspring. Similarly, prenatal betamethasone administration in sheep has been shown to program an increase in $M c 2 r$ mRNA expression in purified adrenocortical cells taken from 1.5-year-old offspring (Su et al. 2013). Taken together, it is likely that alterations in MC2R may contribute to glucocorticoidinduced adrenal deficits. Although Cyp11a1 expression was reduced in male offspring at both 6 and 12 months of age, no other genes were affected by prenatal treatment at any of the time points investigated. We have previously demonstrated that early dexamethasone administration in the sheep results in reduced Cyp11a1 gene expression

Published by Bioscientifica Ltd 
during foetal development (Moritz et al. 2002). CYP11A1 is expressed in all three zones of the adrenal cortex where it is localised to the inner mitochondrial membrane and converts cholesterol into pregnenolone as a precursor of all adrenal hormones. ACTH regulates CYP11A1 expression within the zona fasciculata to produce glucocorticoids, whereas angiotensin II regulates CYP11A1 in the zona glomerulosa to stimulate the production of aldosterone (Hu et al. 1999). At 6 months of age, CYP11A1 is predominantly localised within the zona fasciculata. It is therefore possible that the decrease in Cyp11a1 mRNA in Cort-exposed male offspring from 6 months onwards, may have been caused by negative feedback and also contributed to returning plasma Cort concentrations to control levels by 12 months of age. Of particular interest, at 12 months of age, the pattern of CYP11A1 protein localisation was affected by prenatal Cort exposure. CYP11A1 is predominantly expressed in the zona fasciculata in Untr animals but is expressed predominantly in the zona glomerulosa in Cort-exposed offspring. This may suggest a phenotype more closely associated with altered aldosterone production in later life. These findings are of significant interest considering that we have previously demonstrated increased plasma aldosterone concentrations and reduced renal levels of angiotensin II in the same 6-month-old male offspring that were used in this study. In these animals, we also identified increased expression of glucocorticoidmetabolising enzymes and mineralocorticoid-regulated pathways suggesting important interactions between the programmed adrenal outcomes identified in this study and renal functions identified previously. It is likely that these renal outcomes (Cuffe et al. 2016) contributed to the adrenal changes and increased plasma Cort levels at 6 months identified in this study.

Although prenatal Cort exposure induced changes in adrenal weights, morphology and gene expression at multiple time points throughout the life of male offspring, the adrenal glands of female offspring were not affected by this prenatal perturbation. These sexspecific findings are in accordance with previous findings in dexamethasone studies (Waddell et al. 2010) where programmed disease outcomes in male offspring are frequently more severe than those in female offspring (Moritz et al. 2010). Although these outcomes may be due to sex-specific foetal adaptations to a maternal perturbation, they may also be attributed to differences in the postnatal sex hormone profile, with oestrogen potentially playing a protective role in many programmed disease outcomes (Ojeda et al. 2007).

\section{Conclusion}

Prenatal glucocorticoid exposure can impair the prenatal development of key organs and structures such that offspring are predisposed to dysregulated organ morphology and related physiological outcomes. This study is the first to demonstrate that maternal exposure to corticosterone induces sex-specific physiological and morphological changes in the adrenal gland across the lifespan of the animal. The Cort-induced adrenal outcomes in offspring are age dependant, which highlights the adaptive capacity of the adrenal gland throughout life. These adaptations have resulted in increased basal plasma Cort concentrations in offspring at 6 months of age but not at other time points. Given that high levels of plasma glucocorticoids during adult life can result in impaired renal and cardiovascular physiology, this programmed increase in adrenal glucocorticoid production at 6 months of age may have contributed to the offspring phenotype previously reported (O'Sullivan et al. 2015). Indeed, this study indicates a key role of the adrenal gland in the programming of cardiovascular and renal disease outcomes.

\section{Declaration of interest}

The authors declare that there is no conflict of interest that could be perceived as prejudicing the impartiality of the research reported.

\section{Funding}

This research was funded by the University of Queensland, Australia. K M Moritz was supported by a NHRMC fellowship.

\section{Author contribution statement}

J S M C and K M M were responsible for the conception and design of the experiments. J S M C, E L T, L K A and H B O were responsible for the collection of data. All authors were involved in the analysis and interpretation of the data. All authors were involved in drafting the manuscript and revising it critically for intellectual content.

\section{Acknowledgements}

The authors would like to acknowledge Danielle Burgess, Emily Dorey, Anselm Koning, Lee O'Sullivan and Reetu Singh for assistance with animal breeding and tissue collection.

\section{References}

Anagnostis P, Athyros VG, Tziomalos K, Karagiannis A \& Mikhailidis DP 2009 Clinical review: the pathogenetic role of cortisol in the metabolic syndrome: a hypothesis. Journal of Clinical Endocrinology and Metabolism 94 2692-2701. (doi:10.1210/jc.2009-0370)

Banjanin S, Kapoor A \& Matthews SG 2004 Prenatal glucocorticoid exposure alters hypothalamic-pituitary-adrenal function and blood

Published by Bioscientifica Ltd 
pressure in mature male guinea pigs. Journal of Physiology $\mathbf{5 5 8}$ 305-318. (doi:10.1113/jphysiol.2004.063669)

Bielohuby M, Herbach N, Wanke R, Maser-Gluth C, Beuschlein F, Wolf E \& Hoeflich A 2007 Growth analysis of the mouse adrenal gland from weaning to adulthood: time- and gender-dependent alterations of cell size and number in the cortical compartment. American Journal of Physiology: Endocrinology and Metabolism 293 E139-E146. (doi:10.1152/ajpendo.00705.2006)

Chatelain A \& Dupouy JP 1981 Activity of the pituitary-adrenal system in rat fetuses subjected to encephalectomy in early or late stages of pregnancy. Neuroendocrinology 33 148-152. (doi:10.1159/000123220)

Cheong JN, Cuffe JS, Jefferies AJ, Anevska K, Moritz KM \& Wlodek ME 2016 Sex-specific metabolic outcomes in offspring of female rats born small or exposed to stress during pregnancy. Endocrinology en20161335. (doi:10.1210/en.2016-1335)

Chida D, Nakagawa S, Nagai S, Sagara H, Katsumata H, Imaki T, Suzuki H, Mitani F, Ogishima T, Shimizu C, et al. 2007 Melanocortin 2 receptor is required for adrenal gland development, steroidogenesis, and neonatal gluconeogenesis. PNAS 104 18205-18210. (doi:10.1073/ pnas.0706953104)

Cole TJ, Blendy JA, Monaghan AP, Krieglstein K, Schmid W, Aguzzi A, Fantuzzi G, Hummler E, Unsicker K \& Schutz G 1995 Targeted disruption of the glucocorticoid receptor gene blocks adrenergic chromaffin cell development and severely retards lung maturation. Genes and Development 9 1608-1621. (doi:10.1101/gad.9.13.1608)

Cottrell EC \& Seckl JR 2009 Prenatal stress, glucocorticoids and the programming of adult disease. Frontiers in Behavioral Neuroscience 3 19. (doi:10.3389/neuro.08.019.2009)

Cuffe JS, O'Sullivan L, Simmons DG, Anderson ST \& Moritz KM 2012 Maternal corticosterone exposure in the mouse has sex-specific effects on placental growth and mRNA expression. Endocrinology 153 5500-5511. (doi:10.1210/en.2012-1479)

Cuffe JS, Walton SL, Singh RR, Spiers JG, Bielefeldt-Ohmann H, Wilkinson L, Little MH \& Moritz KM 2014 Mid- to late term hypoxia in the mouse alters placental morphology, glucocorticoid regulatory pathways and nutrient transporters in a sex-specific manner. Journal of Physiology 592 3127-3141. (doi:10.1113/jphysiol.2014.272856)

Cuffe JS, Burgess DJ, O’Sullivan L, Singh RR \& Moritz KM 2016 Maternal corticosterone exposure in the mouse programs sex-specific renal adaptations in the renin-angiotensin-aldosterone system in 6-month offspring. Physiological Reports 4 e12754. (doi:10.14814/phy2.12754)

de Vries A, Holmes MC, Heijnis A, Seier JV, Heerden J, Louw J, WolfeCoote S, Meaney MJ, Levitt NS \& Seckl JR 2007 Prenatal dexamethasone exposure induces changes in nonhuman primate offspring cardiometabolic and hypothalamic-pituitary-adrenal axis function. Journal of Clinical Investigation 117 1058-1067. (doi:10.1172/JCI30982)

Dillberger JE, Cronin NS \& Carr GJ 1992 Prednisone is not a mouse carcinogen. Toxicologic Pathology 20 18-26. (doi:10.1177/019262339 202000103)

Dunn TB 1970 Normal and pathologic anatomy of the adrenal gland of the mouse, including neoplasms. Journal of the National Cancer Institute 44 1323-1389. (doi:10.1093/jnci/44.6.1323)

Ferreira JG, Cruz CD, Neves D \& Pignatelli D 2007 Increased extracellular signal regulated kinases phosphorylation in the adrenal gland in response to chronic ACTH treatment. Journal of Endocrinology 192 647-658. (doi:10.1677/joe.1.06961)

Frith CH 1983 Lipogenic pigmentation, adrenal cortex, mouse. In Endocrine System, pp 60-64. Eds TC Jones, U Mohr, RD Hunt \& CC Capen. Berlin, Heidelberg, Germany: Springer Berlin Heidelberg. (doi:10.1007/978-3-642-96720-7_15)

Garcia-Caceres C, Lagunas N, Calmarza-Font I, Azcoitia I, Diz-Chaves Y, Garcia-Segura LM, Baquedano E, Frago LM, Argente J \& Chowen JA 2010 Gender differences in the long-term effects of chronic prenatal stress on the HPA axis and hypothalamic structure in rats. Psychoneuroendocrinology 35 1525-1535. (doi:10.1016/j. psyneuen.2010.05.006)
Hu MC, Chou SJ, Huang YY, Hsu NC, Li H \& Chung BC 1999 Tissue-specific, hormonal, and developmental regulation of SCC-LacZ expression in transgenic mice leads to adrenocortical zone characterization. Endocrinology 140 5609-5618. (doi:10.1210/ en.140.12.5609)

Huxley RR, Shiell AW \& Law CM 2000 The role of size at birth and postnatal catch-up growth in determining systolic blood pressure: a systematic review of the literature. Journal of Hypertension 18 815-831. (doi:10.1097/00004872-200018070-00002)

Kapoor A, Leen J \& Matthews SG 2008 Molecular regulation of the hypothalamic-pituitary-adrenal axis in adult male guinea pigs after prenatal stress at different stages of gestation. Journal of Physiology 586 4317-4326. (doi:10.1113/jphysiol.2008.153684)

Langdown ML, Smith ND, Sugden MC \& Holness MJ 2001 Excessive glucocorticoid exposure during late intrauterine development modulates the expression of cardiac uncoupling proteins in adult hypertensive male offspring. Pflügers Archiv: European Journal of Physiology 442 248-255. (doi:10.1007/s004240100519)

Leret ML, Peinado V, Gonzalez JC, Suarez LM \& Rua C 2004 Maternal adrenalectomy affects development of adrenal medulla. Life Science 74 1861-1867. (doi:10.1016/j.lfs.2003.07.050)

Moritz K, Butkus A, Hantzis V, Peers A, Wintour EM \& Dodic M 2002 Prolonged low-dose dexamethasone, in early gestation, has no long-term deleterious effect on normal ovine fetuses. Endocrinology 143 1159-1165. (doi:10.1210/endo.143.4.8747)

Moritz KM, Cuffe JS, Wilson LB, Dickinson H, Wlodek ME, Simmons DG \& Denton KM 2010 Review: sex specific programming: a critical role for the renal renin-angiotensin system. Placenta 31 (Supplement) S40-S46. (doi:10.1016/j.placenta.2010.01.006)

O'Sullivan L, Cuffe JS, Koning A, Singh RR, Paravicini TM \& Moritz KM 2015 Excess prenatal corticosterone exposure results in albuminuria, sex-specific hypotension, and altered heart rate responses to restraint stress in aged adult mice. American Journal of Physiology: Renal Physiology 308 F1065-F1073. (doi:10.1152/ajprenal.00676.2014)

Ojeda NB, Grigore D, Robertson EB \& Alexander BT 2007 Estrogen protects against increased blood pressure in postpubertal female growth restricted offspring. Hypertension 50 679-685. (doi:10.1161/ HYPERTENSIONAHA.107.091785)

Ozanne SE 2001 Metabolic programming in animals. British Medical Bulletin 60 143-152. (doi:10.1093/bmb/60.1.143)

Quinn TA, Ratnayake U, Castillo-Melendez M, Moritz KM, Dickinson H \& Walker DW 2014 Adrenal steroidogenesis following prenatal dexamethasone exposure in the spiny mouse. Journal of Endocrinology 221 347-362. (doi:10.1530/JOE-13-0514)

Raber J, Akana SF, Bhatnagar S, Dallman MF, Wong D \& Mucke L 2000 Hypothalamic-pituitary-adrenal dysfunction in Apoe(-/-) mice: possible role in behavioral and metabolic alterations. Journal of Neuroscience 20 2064-2071.

Rosol TJ, Yarrington JT, Latendresse J \& Capen CC 2001 Adrenal gland: structure, function, and mechanisms of toxicity. Toxicologic Pathology 29 41-48. (doi:10.1080/019262301301418847)

Schardein JL, Patton GR \& Lucas JA 1967 The microscopy of 'brown degeneration' in the adrenal gland of the mouse. Anatomical Record 159 291-309. (doi:10.1002/ar.1091590307)

Singh RR, Cuffe JS \& Moritz KM 2012 Short- and long-term effects of exposure to natural and synthetic glucocorticoids during development. Clinical and Experimental Pharmacology and Physiology 39 979-989. (doi:10.1111/1440-1681.12009)

Spiers JG, Chen H-JC, Cuffe JSM, Sernia C \& Lavidis NA 2016 Acute restraint stress induces rapid changes in central redox status and protective antioxidant genes in rats. Psychoneuroendocrinology 67 104-112. (doi:10.1016/j.psyneuen.2016.02.005)

Su Y, Carey LC, Rose JC \& Pulgar VM 2013 Antenatal glucocorticoid exposure enhances the inhibition of adrenal steroidogenesis by leptin in a sex-specific fashion. American Journal of Physiology: Endocrinology and Metabolism 304 E1404-E1411. (doi:10.1152/ajpendo.00013.2013)

Published by Bioscientifica Ltd. 
Sugimoto M, Kojima A \& Endo H 1976 Role of glucocorticoids in terminal differentiation and tissue specific function. Development Growth and Differentiation 18 319-327. (doi:10.1111/j.1440169X.1976.00319.x)

Valenzuela OA, Jellyman JK, Allen VL, Holdstock NB \& Fowden AL 2016 Effects of maternal dexamethasone treatment on pancreatic beta cell function in the pregnant mare and postnatal foal. Equine Veterinary Journal [in press]. (doi:10.1111/evj.12560)

Van Cauwenberge JR, Hustin J, Demey-Ponsart E, Sulon J, Reuter A, Lambotte R \& Franchimont P 1987 Changes in fetal and maternal blood levels of prolactin, cortisol, and cortisone during eutocic and dystocic childbirth. Hormone Research 25 125-131. (doi:10.1159/000180643)

Waddell BJ, Bollen M, Wyrwoll CS, Mori TA \& Mark PJ 2010

Developmental programming of adult adrenal structure and steroidogenesis: effects of fetal glucocorticoid excess and postnatal dietary omega-3 fatty acids. Journal of Endocrinology 205 171-178. (doi:10.1677/JOE-09-0459)

Zilio M, Barbot M, Ceccato F, Camozzi V, Bilora F, Casonato A, Frigo AC, Albiger N, Daidone V, Mazzai L, et al. 2014 Diagnosis and complications of Cushing's disease: gender-related differences. Clinical Endocrinology 80 403-410. (doi:10.1111/cen.12299)

Received in final form 9 October 2016

Accepted 17 October 2016

Accepted Preprint published online 17 October 2016
Published by Bioscientifica Ltd. 